\title{
PLANTEAMIENTO DEL PROBLEMA DE INVESTIGACIÓN: ERRORES DE LA LECTURA SUPERFICIAL DE LIBROS DE TEXTO DE METODOLOGÍA
}

\author{
Alberto Quintana P. ${ }^{1,2}$ \\ Universidad Nacional Mayor de San Marcos, Perú \\ (RECIBIDO EL 7/01/2008, ACEPTADO EL 29/05/2008)
}

\begin{abstract}
RESUMEN
Luego de acentuar la importancia de la investigación en las sociedades modernas y la alarmante desproporción existente en la educación superior peruana entre el número de egresados y el número de tesis sustentadas para la obtención del grado académico, se resalta la importancia de la etapa del "planteamiento del problema de investigación" en la metodología de investigación cuantitativa. Para luego realizar una reflexión conceptual acerca de la etapa referida a partir de dos de los libros de texto de metodología de investigación más usados en nuestro medio, el de Kerlinger y el de Hernández y Batista, con el objetivo de derivar de dicha reflexión un conjunto de sugerencias procedimentales que orienten a los investigadores acerca de las acciones más recomendables para realizar un eficaz Planteamiento del Problema de investigación. Para terminar se citan algunas pautas genéricas para la redacción de un articulo de investigación y se sugiere al lector profundizar estos conocimientos en manuales como Publication manual of the American Psychological Association o La rédaction scientifique.
\end{abstract}

Palabras clave: Tesis de grado, Investigación cuantitativa, Planteamiento del problema, Redacción científica.

\begin{abstract}
After of emphasizing the importance of the investigation in the modern societies and the alarming existing disproportion in the Peruvian education superior between the number of withdrawn and the number of theses sustained for the obtaining of the academic degree, the importance of the stage of the "exposition of the problem of investigation" in the methodology of quantitative investigation is emphasized. Soon to realise a conceptual reflection about the stage referred from two of text books of used methodology of investigation more in our means, the one of Kerlinger and the one of Hernandez and Batista, with the aim of deriving from this reflection a set of procedural suggestions that orient to the investigators about the most recommendable actions to realise an effective Exposition of the Problem of investigation. In order to finish they mention themselves some you rule generic for the writing of an

1 Docente Principal de la Facultad de Psicología de la Universidad Nacional Mayor de San Marcos. E-mail: albertolqp@yahoo.es

2 Se agradece la colaboración en la redacción de esta entrega a Miguel Lerzundi estudiante de la maestría en Psicología Clínica y de la Salud de Universidad Nacional Mayor de San Marcos.
\end{abstract}




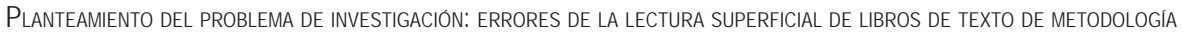

investigation article and the reader to deepen these knowledge in manuals like Publication manual of the American Psychological Association or The scientific drafting.

Keywords: Thesis of degree, quantitative investigation, exposition of the problem, scientific writing.

"La formulación de un problema es muchas veces más importante que la solución, la cual puede ser meramente una cuestión de habilidad matemática o experimental"

Albert Einstein

\section{INTRODUCCIÓN}

Dada la constatación que "El rol de la investigación, la innovación y la generación de tecnologías es hoy el motor central del crecimiento y de la dinámica económica de las sociedades modernas" (Rama, 2007) y la alarmante desproporción existente en la educación superior peruana entre el número de egresados y el número de tesis sustentadas para la obtención del grado académico tanto en el pregrado como en el postgrado (Abeledo, C., 2003), y, más aún, cuando se observa que las tesis desarrolladas muestran evidentes limitaciones desde el Planteamiento del problema de investigación (Zamalloa, E. 2004; Quintana, 2006). Etapa que, al decir de los expertos, como lo enfatiza la cita de Einstein, "es muchas veces más importante que la solución”, o, dicho de otra manera, "un problema correctamente planteado está parcialmente resuelto ..." (Aekoff, 1953, citado por Hernández y Batista, 2003, p. 10), resulta pertinente realizar una reflexión conceptual, y derivar de ella un conjunto de sugerencias procedimentales, que orienten a los investigadores acerca de las acciones más recomendables para realizar un eficaz planteamiento del problema de investigación; orientaciones que se espera coadyuven a la repotenciación de la investigación en nuestro medio.

Una forma adecuada de iniciar la reflexión acerca del tema en cuestión implica el análisis de lo que postulan dos de los libros de texto de metodología de Investigación más usados y empoderados en nuestro medio a este respecto, a saber el de: Kerlinger (2000); y el de Hernández y Batista (2003). La forma cómo se presenta la descripción de esta etapa de investigación en los textos mencionados es obviamente derivada del denominado método de investigación hipotético-deductivo, también conocido como investigación cuantitativa, entendido éste como "el conjunto de procedimientos por los cuales a) se plantean los problemas científicos y b) se ponen a prueba las hipótesis científicas" (Bunge, 1981, p. 55). Y que, en consecuencia, lo que se afirma en ellos no es generalizable a la etapa análoga que se deriva del denominado método de investigación inductivo-conceptual, también conocido como investigación cualitativa, el cual tiene un propósito y se sustenta en una lógica marcadamente distinta (Mejía, 2003, Quintana 2006).

Kerlinger (2000), al abordar este tema, se pregunta y responde “¿En qué consiste el buen planteamiento del problema? Aun cuando los problemas de investigación difieren en gran medida y no hay una forma "correcta" para su planteamiento, algunas de sus características y planteamientos se pueden aprender a utilizar con provecho. Por lo pronto, 
se hará referencia a dos o tres ejemplos de problemas de investigación publicados, y se estudiarán al mismo tiempo sus características... Un problema, entonces, es una oración o aseveración interrogativa en la cual se pregunta: ¿qué relación existe entre dos o más variables?" (p. 18).

Obsérvese, la respuesta que da este autor no corresponde a su pregunta. Y que la pregunta no era ¿Qué es un Problema de Investigación?, si bien incluso su respuesta a esta última pregunta podría ser cuestionada por una serie de consideraciones conceptuales, basta para el fin propuesto en esta entrega el haber explicitado que el autor no ha respondido la pregunta: ¿En qué consiste el procedimiento de plantear el problema? En tanto que el fin propuesto es derivar sugerencias procedimentales que orienten a los investigadores acerca de las acciones más recomendables para realizar un eficaz Planteamiento del Problema,

A este respecto también Hernández; Fernández y Baptista (2003), se preguntan y responden “¿Qué es plantear el problema de investigación?... En realidad, plantear el problema no es sino afinar y estructurar más formalmente la idea de investigación. El paso de la idea al planteamiento del problema puede ser en ocasiones inmediato, casi automático, o bien llevar una considerable cantidad de tiempo; lo que depende de qué tan familiarizado esté el investigador con el tema a tratar, la complejidad misma de la idea, la existencia de estudios antecedentes, el empeño del investigador y las habilidades personales de éste. El seleccionar un tema, una idea, no coloca inmediatamente al investigador en una posición que le permita comenzar a considerar qué información habrá de recolectar, por qué métodos y cómo analizará los datos que obtenga. Antes necesita formular el problema específico en términos concretos y explícitos y de manera que sea susceptible de ser investigado por procedimientos científicos (Selltiz et aL, 1976)." (p. 10). (El subrayado es del texto)

Nótese, si bien los autores utilizan una cita textual de Selltiz para responder su pregunta; sin embargo, en tanto a) el libro de este autor data de hace tres décadas y existe la tendencia a suponer que lo más actual es necesariamente mejor; b) que los autores citados son quienes han resaltado en cursiva el concepto que consideran principal en el texto "afinar y estructurar más formalmente la idea de investigación" (el subrayado es nuestro) y c) que en su texto inmediatamente agregan "Los criterios -de acuerdo con Kerlinger (1973)- para plantear adecuadamente el problema de investigación son: 1) El Problema debe expresar una relación entre dos o más variables, 2) El problema debe estar formulado claramente y sin ambigüedad como pregunta, ....3) El planteamiento implica la posibilidad de una prueba empírica ...". Entonces, considerando que a) Kerlinger no ha respondido adecuadamente a la pregunta en referencia y ellos subrayan en el texto tomado de Selltiz aquello de "afinar y estructurar más formalmente la idea de investigación", no es de extrañar que esta manera de presentar el tema que nos ocupa lleve al investigador novato a confundir el planteamiento del problema de investigación, con la simple verificación de que el problema que tiene la intención de investigar tiene un formato adecuado.

Para ilustrar la confusión a la que nos referimos recurriremos a una analogía con el lenguaje; por ejemplo, a pesar que el enunciado "El Sol gira alrededor de la Tierra" posee una forma bien formada, es decir, está correctamente formulado desde el punto de vista gramatical, sin embargo, es también un enunciado falso. En efecto, el enunciado cumple perfectamente con las reglas de una buena construcción gramatical: está constituido por 
una frase nominal "El Sol" seguida por una frase verbal "gira alrededor de la Tierra", a su vez adecuadamente constituidas; además todos sus elementos son concordantes en género y número, etc. Sin embargo, sabemos, desde Copérnico, que esta evidencia formal no lo hace un enunciado cierto. De la misma manera, un supuesto "Problema de investigación" (sic) puede muy bien ser enunciado con una buena forma, es decir, puede cumplir con las reglas formales, por ejemplo, expresar una relación entre variables, ser una pregunta clara e in-ambigua, implicar la posibilidad de ser contrastado con la realidad (implicar una prueba empírica), y otras reglas o criterios que habitualmente se mencionan en los manuales frecuentemente usados en nuestro medio para la Investigación en Psicología (Kerlinger, 2000; Alarcón, 1999; Hernández y Batista, 2003), y, sin embargo, puede estar mal planteado o simplemente no haber sido planteado.

En este punto, es preciso hacer explícito que en los niveles de investigación exploratorio y descriptivo reconocidos en la investigación cuantitativa es imposible que los problemas de investigación cumplan con el formato propuesto (Kerlinger, 2000; Alarcón, 1999; Hernández y Batista, 2003), ya que por definición, en los niveles de investigación exploratorio y descriptivo aun no se pueden establecer relaciones entre las variables. Es por ello que cuando en los textos de metodología de investigación cuantitativa se describe el formato adecuado que deben tener las preguntas o problemas de investigación (lo que en las disciplinas formales -lógica, matemática- se denominaría una Formula Bien Formada: F.B.F.), sin lugar a dudas se están refiriendo específicamente a los Problemas Bien Formulados en los niveles de investigación descriptivo correlacional o causal.

Recapitulando, consideramos que darle la forma correcta al enunciado de un problema de investigación, es una condición necesaria pero no suficiente para plantear adecuadamente el problema de investigación. La reflexión hecha hasta el momento permite explicar de dónde surgió la tan difundida costumbre existente en nuestro medio de considerar que la función principal de la etapa de planteamiento del problema en un proyecto de investigación, es proponer un enunciado de Problema Bien Formulado. A este respecto, es conveniente explicitar que si bien en los libros de texto de metodología de investigación, en los manuales de divulgación introductorios a la metodología científica, como en cualquier manual, por su propia naturaleza, y presumiblemente por consideraciones didácticas que no viene al caso discutir en este contexto, se abstraen aspectos y características de las acciones constituyentes del método de investigación esquematizándolas y clasificándolas, de suerte que terminan dando la impresión de que se pasa directamente de la etapa de concepción de la idea de investigación a dar el formato correcto al enunciado del problema de investigación, precisándolo y afinándolo (lograr un enunciado de Problema Bien Formulado). Esta confusión parece haberse consolidado debido a que personas bien intencionadas y probablemente competentes en la construcción y manejo de instrumentos de evaluación, o en otros casos, de herramientas estadísticas para el procesamiento de datos, pero novatas en cuanto a los supuestos epistemológicos hipotético-deductivos que fundamentan a la metodología de investigación cuantitativa, a partir de una lectura superficial de estos libros de texto, han cometido el grave error de pasar por alto la actividad más importante del procedimiento de plantear y formular un problema de investigación, a saber: la argumentación razonable por la cual el investigador llega a la conclusión de que en la disciplina científica en la cual contextualiza su estudio existe la necesidad de 
responder a tal interrogante, a tal problema científico. Este error sería grave puesto que si el investigador novato no hace esta argumentación, corre el riesgo de que su estudio no aporte nuevos conocimientos, ni llene un vacío en la estructura de conocimientos de la disciplina, así como tampoco resuelva una contradicción explicitada en los saberes de la disciplina.

En otras palabras, en lugar de plantearse un problema de investigación el investigador novato solamente terminará proponiéndose resolver un problema de aprendizaje personal, de "desconocer" algún tema, en cuyo caso, usualmente, en vez de plantear el problema de investigación, sólo se limitará a exponer algunas anécdotas de las situaciones que despertaron su curiosidad por la temática acerca de la cual se interroga. $\mathrm{O}$ bien, en el mejor de los casos, el investigador novato sólo se limitará a justificar el interés y pertinencia social de dar respuesta a una problemática que demanda una urgente intervención profesional, en cuyo caso, habitualmente en vez de plantear el problema, sólo se limitará a citar documentos institucionales que exponen lineamientos de política sectorial y/o resultados de observaciones epidemiológicas alarmantes que motivaron su interés por la temática acerca de la cual se interroga. Resulta obvio que para resolver satisfactoriamente estas interrogantes no tendría sentido proyectar una investigación científica; por el contrario, para el caso de su "problema de aprendizaje personal", bastaría con tan sólo disponer el acceso a las fuentes de información correspondientes que le permitan aprender los conocimientos existentes a ese respecto en la disciplina y, para el caso del "problema que demanda una urgente intervención profesional”, bastaría con tan sólo disponer la administración de las técnicas e instrumentos de evaluación profesional necesarios, y habitualmente conocidos por un profesional medianamente informado, para efectuar un eficiente diagnóstico situacional específico, preámbulo insoslayable de una intervención profesional sustantiva.

Efectivamente, en el presente documento buscamos recuperar el sentido de la formulación de un problema de investigación como la demostración o argumentación razonable de que existen razones necesarias y suficientes, de acuerdo al estado del conocimiento científico, teórico y empírico vigente en una disciplina, para considerar que aun se desconoce la respuesta a cierta interrogante ${ }^{3}$.

Como consecuencia de las reflexiones anteriores Ud. lector con toda probabilidad habrá aumentado su incertidumbre en relación a ¿qué hacer? para comenzar a elaborar un proyecto de investigación con metodología de investigación hipotético-deductiva, o cuantitativa, por ello una adecuada gestión de esta incertidumbre implica la necesidad de que en las siguientes secciones se recomienden algunas acciones que aseguren un adecuado planteamiento del problema. Estas recomendaciones pretenden ser lo más prácticas y sistemáticas posible, basadas, por supuesto, en la literatura disponible sobre metodología de la investigación científica, y en la experiencia del autor como investigador y como docente de asignaturas referidas a metodología de investigación. Así, se propondrá un conjunto de acciones previas al procedimiento de plantear y formular el problema de investigación, el procedimiento mismo de plantear y formular un problema de investigación que como se verá en su

3 En una entrega futura se buscará, así mismo, recuperar el sentido de la elaboración del Marco Teórico como la demostración o argumentación razonable de que existen razones necesarias y suficientes, de acuerdo al estado de conocimiento científico vigente en una disciplina, para considerar que la respuesta más plausible al problema de investigación planteado es la Hipótesis formulada. 
momento asegura el cumplimiento de los criterios habitualmente recomendados en los libros de texto de metodología de investigación para reconocer si un problema está bien o mal formulado, y por último, algunas acciones y recomendaciones, a partir de los estándares compartidos por la comunidad científica en psicología, para la redacción de este apartado del proyecto de investigación. Insistimos una vez más que reducir la etapa de planteamiento o formulación del problema de investigación, como habitualmente lo hace un investigador novato, a la simple presentación de una pregunta arbitrariamente enunciada verificando que la misma cumple con los criterios habitualmente mencionados en los manuales de divulgación introductorios a la metodología científica, seria entender por Planteamiento del Problema, tan sólo, el enunciar un problema con una "Buena Forma", es decir, con un formato adecuado, en lugar de entender esta etapa de la investigación como el análisis crítico de un conjunto de hechos y conceptos, con el objetivo de descubrir una laguna o inconsistencia en el cuerpo de conocimientos de la disciplina.

\section{ACCIONES PREVIAS AL PLANTEAMIENTO O FORMULACIÓN DEL PROBLEMA DE INVESTIGACIÓN}

Como antes se ha mencionado plantear un problema de investigación es el procedimiento de exponer los argumentos razonables y verdaderos que demuestran que en una disciplina científica determinada aún está pendiente de ser resuelta cierta interrogante, y por ende, que existe la necesidad de proyectar una investigación al respecto. La interrogante en referencia se constituye en el problema de investigación. Por ello resulta lícito afirmar que el problema de investigación debe ser la conclusión lógica de los argumentos expuestos a lo largo del planteamiento del problema y, en consecuencia, frente a la pregunta ¿cómo y por dónde empezar a investigar?, resulta evidente que antes de proceder a plantear el problema son necesarias algunas acciones previas: 1) Concebir la idea a investigar, 2) informarse del contexto de la investigación especializada, 3) precisar los temas susceptibles de ser investigados, y 4) revisar las conceptualizaciones teóricas relacionadas.

\section{Concebir la idea a investigar}

Esta etapa empieza por definir un área temática o seleccionar un campo de trabajo problemático dentro de la especialidad; por ejemplo, consideremos la siguiente información de un diario de circulación nacional:

"En la evaluación nacional de rendimiento del año 2004 sólo $10 \%$ de los egresados de secundaria, en el mejor de los casos, aprobó los reactivos de matemática y un porcentaje similarmente pequeño aprobó los reactivos de comprensión de textos. El gobierno no sabe a ciencia cierta la causa de estos pobres resultados, sobre todo si se consideran los esfuerzos realizados por mejorar la educación pública".

En este caso, un psicólogo podría concebir la idea de investigar los posibles determinantes psicológicos del fracaso escolar.

La condición necesaria para concebir una idea de investigación como la mencionada es estar atento a las ideas que pueden surgir en diferentes contextos. Por ejemplo, en los 
medios de divulgación científica como revistas, documentales, informaciones de medios de comunicación. Supongamos que un psicólogo por ejemplo trabaje en un centro educativo y se encuentre revisando bibliografía sobre los determinantes del aprendizaje escolar, no sería raro que en este ínterin, lea un texto que le recuerde que la calidad de la enseñanza en el aula está relacionada a la motivación del docente. Puede ser también que el germen de la idea haya surgido como en el ejemplo mencionado de informes de medios de divulgación de novedades científicas, leer en el diario, escuchar en la radio o ver en la televisión un reportaje sobre los pobres resultados que se obtuvieron en las pruebas de rendimiento para escolares el año 2004.

De otro lado, el investigador revisando revistas de investigación puede enterarse de un estudio exploratorio realizado para determinar los factores que explican las diferencias de rendimiento entre escuelas con similares recursos económicos, incluso escuelas cercanas dentro de un mismo distrito.

Así mismo, la idea de investigación puede haber surgido en intercambios de ideas con colegas y con especialistas en el tema, por ejemplo en un congreso sobre la problemática de la educación.

El tema de investigación puede surgir, también, a través de la propia experiencia laboral profesional, por ejemplo un psicólogo de una escuela de educación básica regular firmemente convencido en el potencial de autorrealización como personas de los alumnos y que, sin embargo, se enfrenta cotidianamente con problemas de bajo rendimiento escolar, con las dificultades que tienen los alumnos para cumplir con el nivel estándar de rendimiento.

Finalmente, otra fuente importante de ideas de investigación son los documentos institucionales como lineamientos de políticas sectoriales, índices epidemiológicos, etc.

Por cualquiera de estos medios puede hacerse el primer acercamiento al problema. A partir de estas fuentes, es aconsejable elaborar citas contextuales y alguna que otra cita textual que se usarán de insumo durante la redacción de la Formulación del Problema, más específicamente al abordar lo referente a la Justificación de la Investigación.

\section{Informarse del contexto de la investigación especializada}

Esto significa informarse rápida y panorámicamente del contexto de la investigación especializada e identificar las instituciones y los investigadores más actualizados. En el caso de la psicología, esto se realiza revisando la base de datos impresa Psychological abstract o las bases de datos electrónicas especializadas (Psicodoc, Psico Info, Psyc Clip, Med Line, Pro Quest, etc.), las cuales difunden los resúmenes de los artículos publicados en revistas indexadas especializadas de la disciplina, es decir en las revistas de mayor prestigio académico en esa área. Esto permite al investigador una visión global del estado de la investigación en el tema de su interés que le orienta acerca de los problemas específicos que en ese momento ocupan a los investigadores, las instituciones en las que investigan, las revistas que les sirven de medios para comunicar sus hallazgos y los grupos de investigación existentes en los diferentes confines del mundo conocido. Estas fuentes solo deberían usarse, de no tener otra alternativa, para elaborar citas contextuales, 
mas resultaría impropio usarlas para elaborar citas textuales; en tanto por su intermedio solamente se comunican resúmenes o abstracts de los estudios. Resúmenes en los que muchas veces se tiende a omitir algunas referencias metodológicas; necesarias para poder estimar adecuadamente la validez interna y externa de los hallazgos reportados.

\section{Precisar los temas susceptibles de ser investigados}

Por ello se entiende precisar los temas pendientes y, por ende, aún susceptibles de ser investigados en la temática de interés, revisando las publicaciones correspondientes de las instituciones e investigadores, en las revistas especializadas impresas o electrónicas previamente identificadas (para acceder a las citadas publicaciones se recomienda acudir a hemerotecas en unos casos y en otros usar los buscadores de la Web; Altavista, Google, etc.), en los cuales, dado su carácter no especializado, no es conveniente hacer la búsqueda sólo por temas, sino más específicamente por la conjunción de autores y temas. A partir de estas fuentes, sería aconsejable elaborar citas contextuales y alguna que otra cita textual que se usara de insumo obligatorio durante la redacción de la Formulación del Problema.

\section{Revisar las conceptualizaciones teóricas relacionadas}

Este proceso pasa por revisar las conceptualizaciones teóricas relacionadas a la temática de interés que han sido propuestas por investigadores y académicos reconocidos (en textos y libros de la especialidad), es decir académicos cuyos conocimientos han sido validados por la comunidad académica y científica de la especialidad. El acceso a estas fuentes se podrá realizar por intermedio de las bibliotecas de instituciones académicas y de investigación acreditadas e incluso de bibliotecas virtuales (por ejemplo el E-LIBRO). A partir de estas fuentes también sería aconsejable elaborar citas contextuales y alguna que otra cita textual que se usará de insumo obligatorio durante la redacción de la Formulación del Problema

Recién después de haber realizado estas cuatro acciones, que no necesariamente son todas secuenciales, puede decirse propiamente que el investigador se encuentra listo para iniciar el procedimiento de plantear y formular un problema de investigación. Es por ello que Hernández, Fernández y Baptista (1996) inician su respuesta a la interrogante ¿Qué es plantear el problema de investigación? afirmando:

"Una vez que se ha concebido la idea de investigación y el científico, estudiante o experto social han profundizado el tema en cuestión (acudiendo a la bibliografía básica, así como consultando a otros investigadores y fuentes diversas), se encuentran en condiciones de plantear el problema de investigación."(p. 10) 


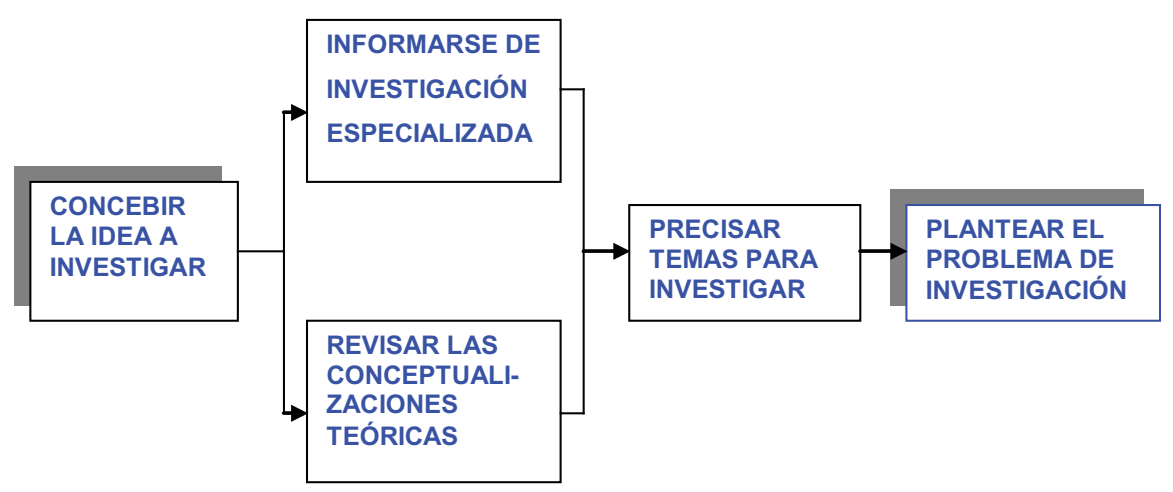

Figura 1. Acciones previas al planteamiento del problema de investigación.

\section{PLANTEAMIENTO Y FORMULACIÓN DEL PROBLEMA DE INVESTIGACIÓN}

Como hemos mencionado, esta etapa significa argumentar la pertinencia académica de formularse determinadas interrogantes respecto al tema elegido; es decir, demostrar, sustentándose en la información recogida por medio de las acciones previas, la existencia de cierta ausencia de conocimiento o inconsistencias teóricas o teórico-empíricas en los conocimientos existentes por el momento respecto al tema de interés en la disciplina en referencia. En ello coincidimos con Bunge (1981) quien, al exponer esquemáticamente el método científico, precisa:

\section{Planteo del problema}

Reconocimiento de los hechos: examen del grupo de hachos, clasificación preliminar y selección de los que probablemente sean relevantes en algún respecto.

Descubrimiento del problema: hallazgo de la laguna o de la incoherencia en el cuerpo del saber.

Formulación del problema: planteo de una pregunta que tiene probabilidad de ser la correcta; esto es, reducción del problema a su núcleo significativo, probablemente soluble y probablemente fructífero, con ayuda del conocimiento disponible.

Para demostrar la existencia de cierta ausencia en los conocimientos, es necesario presentar los conocimientos teóricos y empíricos que existen sobre el tema, elaborando la fundamentación académica que explicite la carencia o limitación de la información acerca de los aspectos que se pretende investigar (refrendados por las citas bibliográficas y hemerográficas especializadas correspondientes). Y, además, justificar la investigación argumentando el interés sociocultural profesional que reviste el tema por ser un lineamiento de política sectorial y/o por la prevalencia de la problemática en referencia (refrendados por las citas recabadas de fuentes como los documentos institucionales o informes técnicos acerca del sector). 
Para demostrar la existencia de inconsistencias teóricas o teórico-empíricas en relación al tema que se pretende investigar, es necesario argumentar presentando teorías o datos empíricos cuyas conclusiones sean contradictorias entre sí, es decir:

- Explicitando la contradicción entre los planteamientos teóricos y la realidad a la que se refieren, por ejemplo: postular que las personas tiene como rasgo de personalidad, entre otros, un patrón de relación interpersonal, autoritario o tímido, que se mantiene constante con independencia de los cambios en las situaciones, afirmación que se contradice con la observación cotidiana de personas que la sabiduría popular califica de "Un león en la oficina y un ratón en su hogar, o viceversa".

- Explicitando la contradicción entre los planteamientos teóricos y hallazgos empíricos, por ejemplo: encontrar que las conclusiones (sic) de ciertas investigaciones empíricas "la simple toma de apuntes de una clase aumenta la comprensión de la misma", parece contradecir determinados postulados teóricos "es imposible que un sistema, o persona, pueda registrar información, o tomar apuntes, de aquello que no ha procesado, o comprendido".

- Explicitando la contradicción entre los planteamientos teóricos de conceptualizaciones alternativas, por ejemplo: en el ámbito de la Psicología Cínica y de la Salud encontrarse, de un lado, con los postulados de una "corriente" psicológica que afirma la existencia de un continuo entre la normalidad, "neurosis" y psicosis, organizado en función de una gradiente de menor a mayor gravedad de "desajuste de la personalidad", y por otro lado, los postulados de otra "corriente" psicológica que afirma que las "neurosis" y las psicosis son ortogonales entre sí, es decir no guardan relación, en tanto serían "desajustes de la personalidad" determinados por diferentes causas. 


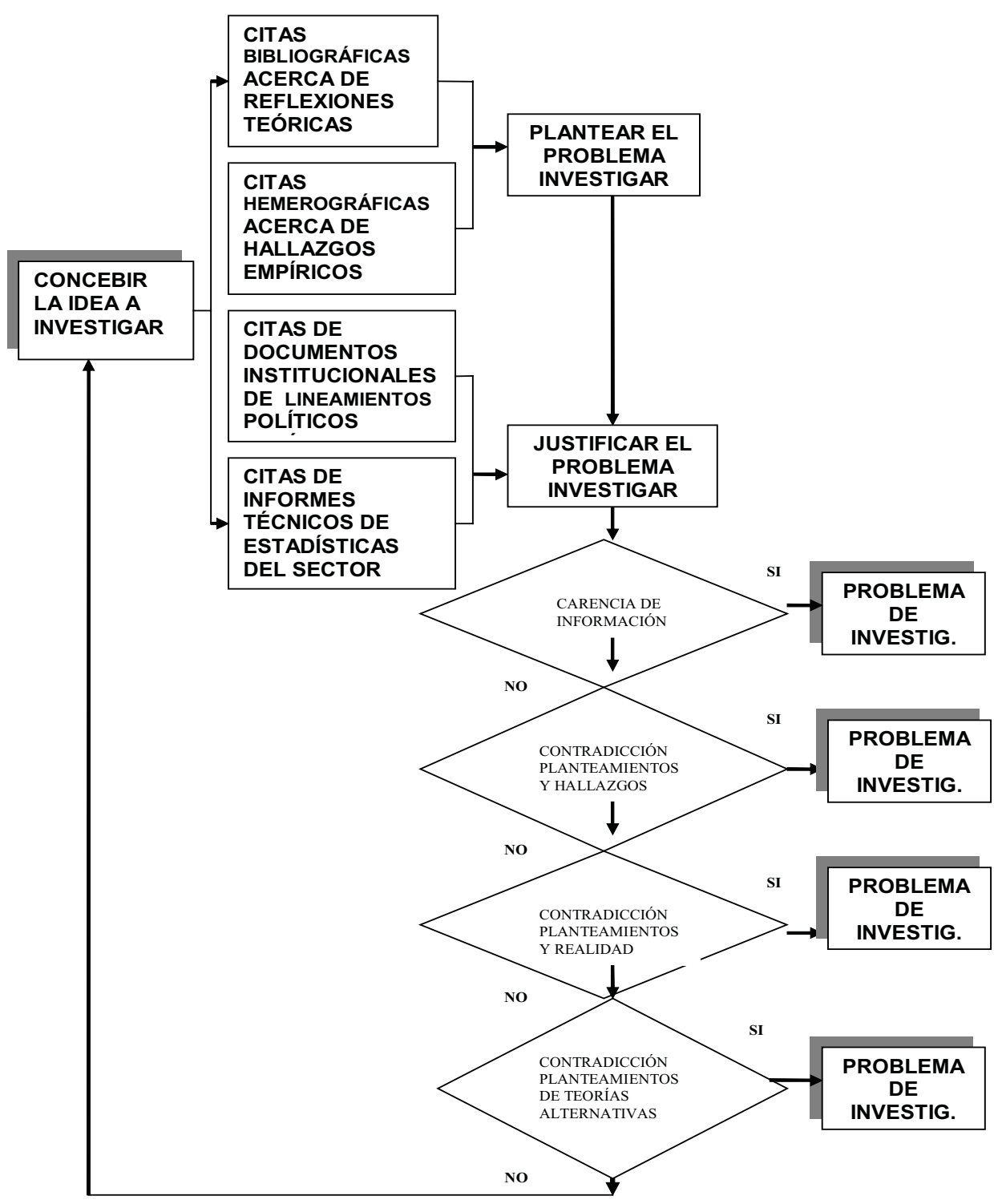

Figura N. ${ }^{\circ}$ 2. Planteamiento del problema.

En conclusión, la formulación del problema consiste entonces en elaborar una argumentación razonable, en el contexto de los datos y las conceptualizaciones aceptadas por la comunidad académica de la especialidad, que explicite una ausencia en los conocimientos existentes o una inconsistencia en los conocimientos existentes respecto al tema elegido. Argumentación que tiene como conclusión necesaria la pregunta o el problema a investigar. 
Recién después de haber explicitado la mencionada argumentación, es pertinente afinar la "Buena Forma" del enunciado del problema, puesto que si hemos realizado una razonable argumentación para demostrar que en determinada disciplina científica aún se encuentra pendiente de ser resuelta cierta interrogante, el problema de investigación, de suyo que un problema de investigación así planteado, cumplirá estrictamente con los criterios habitualmente mencionados en los libros de texto de metodología de investigación para verificar si un Problema está Bien Formulado. Esto ocurre incluso respecto a los criterios pertinentes para los niveles de investigación exploratorio y descriptivo, a saber, que el problema: tendrá significancia, es decir, que su resolución contribuirá a aumentar los conocimientos en el área a investigar. Estará bien delimitado, es decir que el investigador habrá ajustado sus recursos al área donde tiene mayor experiencia. Tendrá originalidad, lo que implica que será novedoso generando interés y entusiasmo en el investigador. Y, por último, establecerá claramente, y sin ambigüedad, la información requerida para resolverlo. De otro lado, si por el estado de los conocimientos en la disciplina acerca de la temática de interés se estuviera en condiciones de plantear un problema de investigación causal o correlacional, presumiblemente este problema además de lo ya mencionado expresará una relación entre dos más variables, permitirá la posibilidad de realizar una prueba empírica del mismo, y tendrá factibilidad, es decir que será factible de ser investigado en las condiciones y en el tiempo previstos. Y también, de suyo que un problema de investigación así concebido, estará bastante alejado de los criterios mencionados en los libros de texto de metodología de investigación para reconocer cuándo un Problema está Mal Formulado, a saber, que sería poco menos que imposible que pueda ser muy amplio o no esté adecuadamente limitado. Tampoco podrá ser muy específico o intrascendente o que no se pueda medir en la práctica. Asimismo, no podría haber sido antes resuelto y haber dejado de representar una novedad. Tampoco sería posible que se trate de un tema filosófico o que esté expresado en términos valorativos. En conclusión, se puede afirmar que un problema de investigación adecuadamente planteado necesariamente sería un enunciado que cumpliría los criterios requeridos en un Problema Bien Formulado.

Recapitulando, es pertinente enfatizar que la elaboración del texto al plantear el problema de investigación deberá explicitar las razones que sustentan la investigación al grado que las ideas postuladas puedan ser verificadas con la revisión de las referencias citadas y además puedan ser conocidas, contrastadas y aprovechadas por otros investigadores; es decir, este componente de la tesis o proyecto de investigación, permítase la redundancia, en el marco de la lógica hipotético-deductiva que subyace a la denominada Metodología de Investigación Cuantitativa, amerita un pormenorizado análisis empírico y conceptual de parte del investigador por cuanto en el resto del documento éste sólo se va a limitar a demostrar que ha sometido su tesis a contrastación estricta con la experiencia controlada, comprobando bien su veracidad o bien su falsedad. Sobre todo teniendo en cuenta que la citada contrastación con la realidad observada se realiza mediante una serie de procedimientos algorítmicos estandarizados para el control de la validez interna y externa de los hallazgos, procedimientos que se describen con cierto detalle incluso en los más modestos manuales de difusión de los diseños experimentales (Sánchez y Reyes, 1990; Mormontoy, 1994), y considerando además que se recurre a técnicas de análisis estadístico, tanto paramétrico como no paramétrico, así mismo estandarizadas y algorítmicas, para el 
análisis de los datos recolectados, y que la mayoría de estas técnicas de análisis estadístico son de simple aplicación si se utiliza el muy conocido Paquete Estadístico para las Ciencias Sociales SPSS (Díaz de Rada, 2002; Garrido, 2002).

De otro lado, se pueden señalar sumariamente las principales acciones que tendrá que realizar el investigador una vez que haya elegido para su redacción el capitulo de Planteamiento del Problema:

a) Establecer los párrafos que van a ser objeto de redacción y que, salvo correcciones ulteriores, son las premisas de la argumentación prevista.

b) Seleccionar, clasificar y utilizar las citas de investigación que contienen la información relevante para elaborar una argumentación razonable que permita explicitar una ausencia o bien una inconsistencia en los conocimientos existentes respecto al tema elegido.

c) Redactar las cuestiones a medida que se va elaborando cada párrafo.

d) Corregir el estilo leyendo detenidamente todo el texto a fin de comprobar el rigor de las argumentaciones, la claridad de la exposición y la coordinación de todas las partes, sin vacíos ni repeticiones. Revisando el contenido y la forma de cada párrafo en la redacción final.

Para terminar, es conveniente mencionar que el lector interesado en conocer cómo redactar eficazmente el planteamiento de un problema de investigación encontrará muy instructivo revisar trabajos dedicados a exponer pormenorizadamente guías y pautas para la redacción de reportes de investigación, como Publication manual of the American Psychological Association (APA, 2001) o La rédaction scientifique (IDRC, 2008).

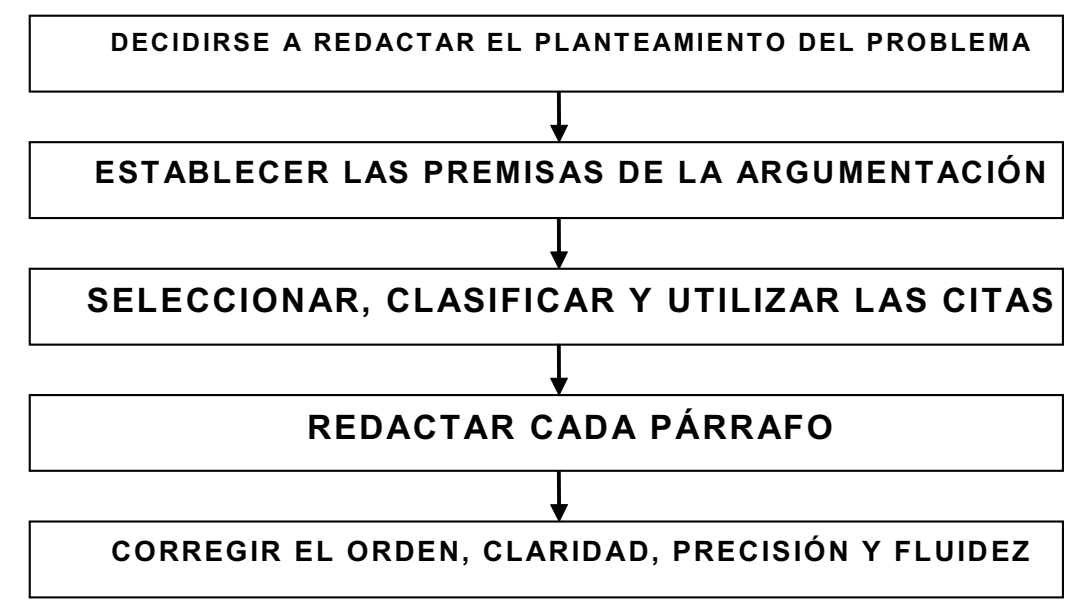

Figura N. ${ }^{o}$ 3. Redacción del planteamiento del problema. 


\section{REFERENCIAS BIBLIOGRÁFICAS}

1. Abeledo, C., (2003). Investigación en universidades y formación de recursos humanos en disciplinas seleccionadas. En: http://www.concytec.gob.pe/ProgramaCyT/ FONCYC/informes/sni_603.pdf. (09/05/08)

2. Alarcón, Reynaldo (1991). Métodos y diseños de investigación del comportamiento. Lima: UPCH.

3. American Psychological Association (APA) (2001). Publication manual of the American Psychological Association. 5a. ed. Washington, D.C.

4. Bunge, Mario (1981). La ciencia, su método y su filosofía. Buenos Aires: Ediciones Siglo XX.

5. Centro internacional de Investigaciones para el Desarrollo (IDRC) (2008). La rédaction scientifique. http://www.idrc.ca/ IMAGES/books//WFC_French//WFC_French/ sitemap.html (09/05/08)

6. Díaz de Rada, V. (2002). Técnicas de análisis multivariante para ilnvestigación social y comercial. Ejemplos prácticos usando el SPSS. Madrid: RA-MA

7. Garrido, G. (2002). SPSS aplicado a las ciencias de la salud. Madrid: RA-MA

8. Hernández, R., Fernández, C. y Baptista P. (1996). Metodología de la investigación científica. México: McGraw-Hill

9. Hernández Sampieri, Roberto; Fernández Collado, Carlos y Baptista Lucio, Pilar (2003). Metodología de la investigación. México: Mc Graw Hill.

10. Kerlinger, Fred (2000). Investigación del comportamiento técnicas y metodología. México: McGraw Hill Interamericana.

11. Martín Benavides y José Rodríguez (2006). Políticas de educación básica 2006- 2011. Lima: CIES.

12. Mejía, J. (2003). "De la construcción del conocimiento social a la práctica de la investigación cualitativa”. En Investigaciones Sociales Rev. del Inst. de Invest. Histórico Sociales Año VII, N. ${ }^{\circ}$ 11, pp. 179-197. Lima: Facultad de Ciencias Sociales, Universidad Nacional Mayor de San Marcos.

13. López Y. J. (1995). La aventura de la investigación científica. Madrid: Edit. Síntesis.

14. Mormontoy (1994). "Elaboración del protocolo de investigación". En Ciencias de la salud, de la conducta y áreas afines. Lima.

15. Quintana A. (2006a). "Análisis Neo-bibliométrico de las Investigaciones de Tesis en la Escuela Académico-Profesional de Psicología UNMSM”. En Revista de Investigación en Psicología vol 9. $\mathrm{N}^{\circ}$ 1. Lima: Instituto de Investigaciones Psicológicas, Facultad de Psicología, Universidad Nacional Mayor de San Marcos.

16. Quintana, A. (2006b). Metodología de investigación cualitativa en Psicología: Tópicos de actualidad. Lima: Facultad de Psicología, Universidad Nacional Mayor de San Marcos, Fondo Editorial. 
17. Rama, C. (2007). Los postgrados en América Latina y el Caribe en la sociedad del conocimiento. México: USUAL.

18. Real Academia Española (1978). Esbozo de una nueva gramática de la lengua española. Madrid: Espasa-Calpe.

19. Sánchez, H. y Reyes, C. (1992). Metodología y diseños de la investigación científica. Lima.

20. Zamalloa, E. (2004). Investigación en Educación Superior. En: http://www.upch. edu.pe/faedu/documentos/din/articulos/zamalloa.pdf. (09/05/08) 